\title{
THYROID DYSFUNCTION IN SLE AND ASSOCIATION OF THYROID ANTIBODY LEVELS WITH DISEASE ACTIVITY IN SLE
}

\author{
Srikantan Sreedharan Pillai', Ganga Velayudhan²
}

${ }^{1}$ Additional Professor, Department of General Medicine, Government Medical College, Thiruvananthapuram. 2Junior Resident, Department of General Medicine, Government Medical College, Thiruvananthapuram.

\begin{abstract}
\section{BACKGROUND}

SLE is the prototype of the autoimmune disease affecting predominantly women. It affects women in their reproductive years. An autoimmune thyroid disease marked by the presence of antibodies directed against thyroid antigens has been associated with SLE, GCA and Sjogren's syndrome. A number of studies have suggested that thyroid disease is more common in SLE. So SLE patients should be routinely investigated for Autoimmune Thyroid disease.

The objectives of this study are to assess thyroid dysfunction and the presence of thyroid antibodies in SLE and to determine the association of thyroid antibodies with disease activity in SLE.
\end{abstract}

\section{MATERIALS AND METHODS}

Patients admitted with SLE in the Department of Medicine, Medical College, Trivandrum during the period 14.5.2010 to 13.1.2010 (six months) were taken up for the study. 100 patients admitted to the hospital with SLE satisfying the inclusion criteria were included in the study. They were subjected to thyroid antibody assay and thyroid function tests. Disease activity was assessed by SLEDAI score.

\section{RESULTS}

Out of 100 patients 26\% had Hypothyroidism, 24\% had Subclinical hypothyroidism and 16\% had hyperthyroidism. 28\% of patients were AMA positive and 39\% were ATG positive. AMA and ATG positivity and SLEDAI Scoring are closely related.

\section{CONCLUSION}

Hypothyroidism was the major thyroid dysfunction in SLE. Positive thyroid antibodies were found to be associated with SLE. There is a close association between SLEDAI scores and positive thyroid antibodies. In addition, there is a statistically significant association between lupus nephritis with vasculitis and positive thyroid antibody levels.

\section{KEYWORDS}

SLE, SLEDAI, ATA, AMA, ATG.

HOW TO CITE THIS ARTICLE: Pillai SS, Velayudhan G. Thyroid dysfunction in SLE and association of thyroid antibody levels with disease activity in SLE. J. Evolution Med. Dent. Sci. 2017;6(33):2684-2688, DOI: 10.14260/Jemds/2017/579

\section{BACKGROUND}

Systemic Lupus Erythematosus (SLE) is a prototype of autoimmune diseases affecting predominantly women in the ratio 9:1. SLE was first described in 1828. ${ }^{1}$ It is characterised by a multisystem organ involvement because of deregulation of self-reactive B cells leading to autoantibody production, immune complex disposition and complement activation with tissue damage. It is important for physicians to be aware of the functional and social consequences of the diseases and to improve the patient's quality of life. When treating a patient with SLE, the aim is to suppress disease activity which is reversible and to prevent the onset of organ damage which is irreversible. This is facilitated by regular care of SLE related conditions.

Financial or Other, Competing Interest: None.

Submission 03-04-2017, Peer Review 16-04-2017,

Acceptance 18-04-2017, Published 24-04-2017.

Corresponding Author:

Dr. Srikantan Sreedharan Pillai,

Department of General Medicine,

Government Medical College,

Thiruvananthapuram.

E-mail: roshnisrikantan@yahoo.co.in

DOI: $10.14260 /$ jemds $/ 2017 / 579$

\section{(c) $(1)(5)$}

It affects predominantly women in their reproductive years. The median age of onset in Indian SLE is 24.5 years and sex ratio (F:M) is 11:11. ${ }^{2}$ Remissions and relapses characterise the disease. The clinical manifestations and their severity in individual patients may vary considerably and therefore the treatment strategy needs to be tailored accordingly.

The American College of Rheumatology has criteria for the classification of patients having SLE. ${ }^{3}$ If the patient has at any time in his or her medical history, 4 of the 11 criteria documented, the diagnosis of SLE can be made with about $95 \%$ specificity and $85 \%$ sensitivity. A number of validated indices are available for quantifying disease activity. The more popular indices is SLEDAI ${ }^{4}$ (SLE disease activity index). They help in formulating the overall treatment plan and assessment of prognosis.

Autoimmune thyroid disease marked by the presence of antibodies directed against thyroid antigens has been associated with a number of non-organ specific rheumatological disorders. ${ }^{5}$ These associations include SLE, Sjogren's syndrome and giant cell arthritis. A number of studies conducted have suggested that thyroid disease is more common in SLE than in the general population, but the disagreement exists as to whether both hypothyroidism and hyperthyroidism are more common or whether this finding is restricted to hyperthyroidism alone. Many SLE patients are 
initially treated for thyroid dysfunction before the diagnosis of lupus is made or vice versa.

Symptoms of thyroid disease can be confused with those of lupus. Although the relationship between autoimmune thyroid disease and SLE has been revealed, the prevalence of thyroid disease in lupus patients is controversial. Reported prevalence of autoimmune thyroid disease and antithyroid antibodies in SLE patients varied considerably. Both antithyroglobulin and antimicrosomal (Antithyroid peroxidase) antibodies have been found with greater frequency in SLE than in general population, even in lupus patients who do not have clinical thyroid disease. ${ }^{6}$ It is still a subject of discussion as to whether SLE is an independent risk factor for these thyroid abnormalities or whether this is a coincidental finding because the group most at risk for SLE, young-to-middle aged women, is precisely the same group most at risk for autoimmune thyroid.

So SLE patients should routinely be investigated for autoimmune thyroid disease. The presence of autoantibodies suggests autoimmunity to be a basis for thyroid dysfunction in SLE.

The prognosis of SLE is quite grim with more than half of the patients developing irreversible organ damage over time. Although, the survival has improved in the west with modern treatment to the tune of $80 \%$ at 10 years after diagnosis, the Indian figures are not good ( $50 \%-60 \%$ survival at 10 years).

\section{MATERIALS AND METHODS}

The study was conducted in the Department of Medicine, Govt. Medical College, Trivandrum. This was a hospital-based cross-sectional study done over a period of 6 months.

Kumar et $\mathrm{al}^{7}$ in their study of 100 SLE patients had reported a prevalence of thyroid dysfunction in 36\% of patients. Based on this, sample size was determined using $\alpha .05$ and error margin $25 \%$ of the observation. Sample size was estimated using the formula $4 \mathrm{XPQ} / \mathrm{l}^{2}$.

Where $\mathrm{P}=$ Prevalence, $\mathrm{Q}=100-\mathrm{P}$ and $\mathrm{l}=$ error margin

The sample size obtained is 113 .

In this study, 100 patients admitted to the hospital with SLE during the period 14.5.2010 to 13.1.2010 (six months) satisfying the inclusion criteria were taken up.

\section{Inclusion Criteria}

Diagnosed patients with SLE.

\section{Exclusion Criteria}

a. Patients with known thyroid dysfunction.

b. Patients on drugs affecting thyroid function.

c. Pregnant females.

Data collection was based on patient demography, symptoms and signs, criteria for diagnosis of SLE and relevant laboratory tests.

\section{Laboratory Tests Include}

1. Measurement of antimicrosomal antibody (AMA) and antithyroglobulin antibody (ATG) by ELISA.

2. Other tests- blood routine, DCT, LDH, urine albumin microscopy, 24-hour urine protein, renal function test, thyroid function test, liver function tests, ANA, AntidsDNA, Renal biopsy.

3. SLEDAI Score

\section{Classification Criteria for Diagnosis of SLE}

Malar Rash- fixed erythema, flat or raised over the malar eminences.

Discoid Rash- Erythematous circular raised patches with adherent keratotic scaling and follicular plugging; atrophic scarring may occur.

Photosensitivity- Exposure to ultraviolet light cause rash.

Oral Ulcers- includes oral and nasopharyngeal ulcers, observed by physician.

Arthritis- Non-erosive arthritis of two or more peripheral joints with tenderness, swelling or effusion.

Serositis- Pleuritis or pericarditis documented by ECG or rub or evidence of effusion.

Renal Disorder- Proteinuria $>0.5 \mathrm{~g} / \mathrm{d}$ or $3+$ or cellular casts.

Neurologic Disorder- Seizures or psychosis without other causes.

Haematologic Disorder- Haemolytic anaemia or leukopenia (<4000/L) or lymphopaenia $(<1500 / \mathrm{L})$ or thrombocytopenia $(<100,000 / \mathrm{L})$ in the absence of offending drugs.

Immunologic Disorder- Anti-dsDNA, anti-Sm and/or antiphospholipid.

Antinuclear Antibodies- An abnormal titre of ANA by immunofluorescence or an equivalent assay at any point in time in the absence of drugs known to induce ANAs.

Any combination of 4 or more of 11 criteria, well documented at any time during patient's history, makes it likely that the patient has SLE (specificity and sensitivity are $95 \%$ and $75 \%$ respectively).

SLEDAI score was used to determine disease activity in SLE. It consists of a maximum score of 105 . The scores are scaled as no activity (0), mild activity $(1-5)$, moderate activity (6 - 10), high activity (11 - 19) and very high activity $(>20)$.

Data were collected using proforma and analysed using statistical programme, percentage sampling for quantitative analysis and statistics package for Social Sciences, SPSS (Version 11) for qualitative analysis to derive the values of probability ( $p$ value), Fisher exact test and Mann-Whitney U test. Results were considered significant at $p$ value $<0.05$.

\section{RESULTS}

Out of 100 patients $41 \%$ were $<30$ yrs., $39 \%$ belonged to 30 39 yrs. of age and $20 \%$ were $>40$ yrs. of age. Of these, $83 \%$ were females and $17 \%$ were males. In the 100 patients studied $26 \%$ had hypothyroidism, $24 \%$ subclinical hypothyroidism and 16\% hyperthyroidism (Fig. 1). Of the 100 patients studied, $28 \%$ were AMA positive (Fig. 2) and $39 \%$ were ATG positive (Fig. 3). Based on SLEDAI score, $41 \%$ had moderate activity, $26 \%$ high activity, $18 \%$ very high and $12 \%$ mild activity. 

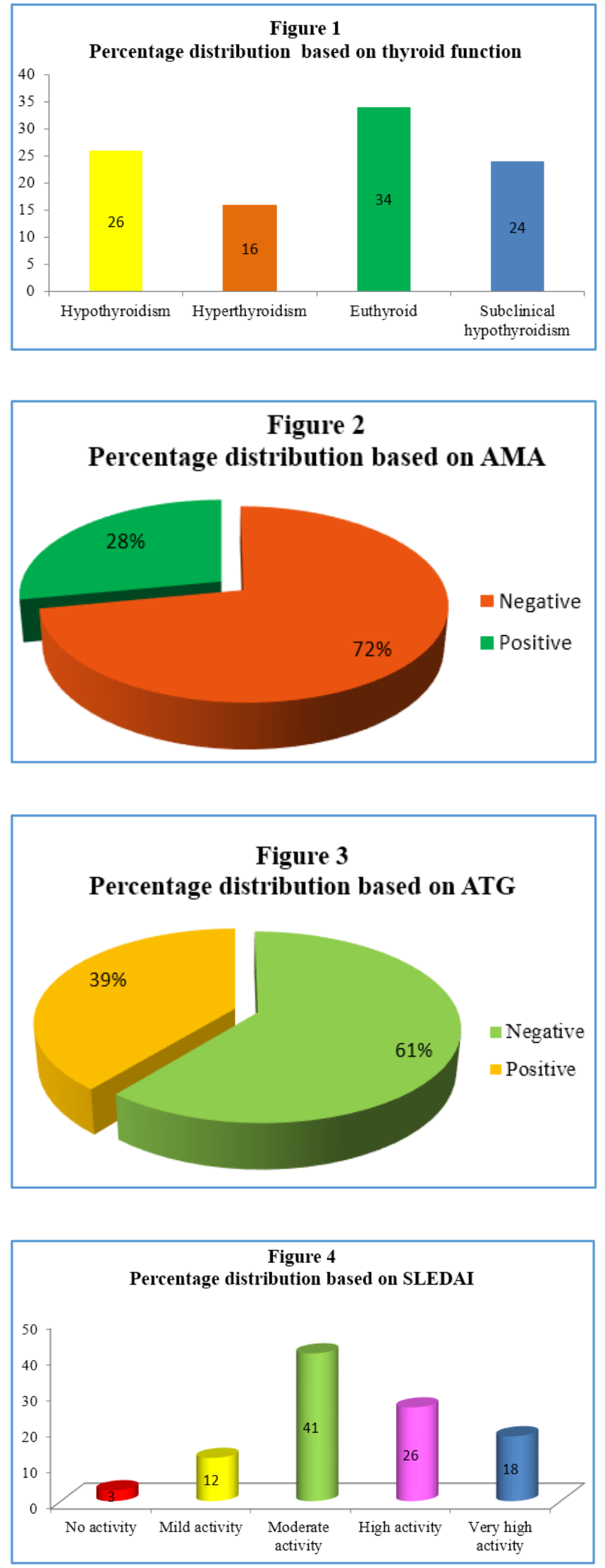

Based on clinical features $73 \%$ had arthritis, $62 \%$ had malar rash, $27 \%$ had oral ulcers and $27 \%$ of patients had serositis. Among the haematological manifestations $12 \%$ had anaemia, $6 \%$ had haemolytic anaemia, 20\% had thrombocytopenia and $11 \%$ pancytopenia. Anaemia with thrombocytopenia was present in $11 \%$, leucocytosis was present in $6 \%$.
Lupus nephritis was present in $37 \%$ of patients (Fig. 5). Neurological manifestations were seen in $38 \%$ of patients, of which $17 \%$ had seizures. Vasculitis was present in $8 \%$, alopecia in $15 \%$ and thyromegaly in $21 \%$; $43 \%$ had photosensitivity.

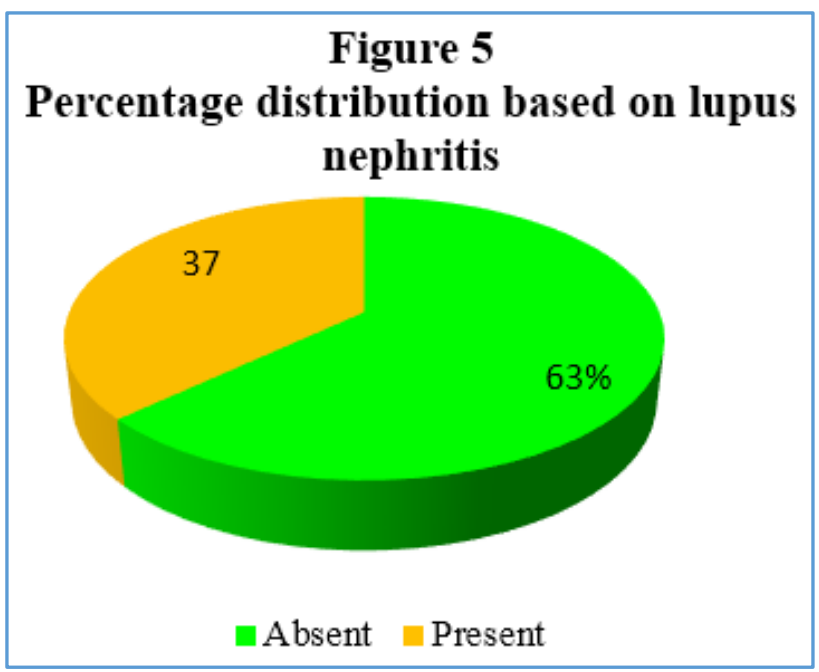

Based on serology $79 \%$ were both ANA and anti-dsDNA positive, $17 \%$ were ANA positive and $2 \%$ anti-dsDNA positive. There is a close relationship between AMA and ATG positivity and SLEDAI scoring (Fig. 6 and Fig. 7). There was increase in the SLEDAI score with increasing values of antibody positivity.

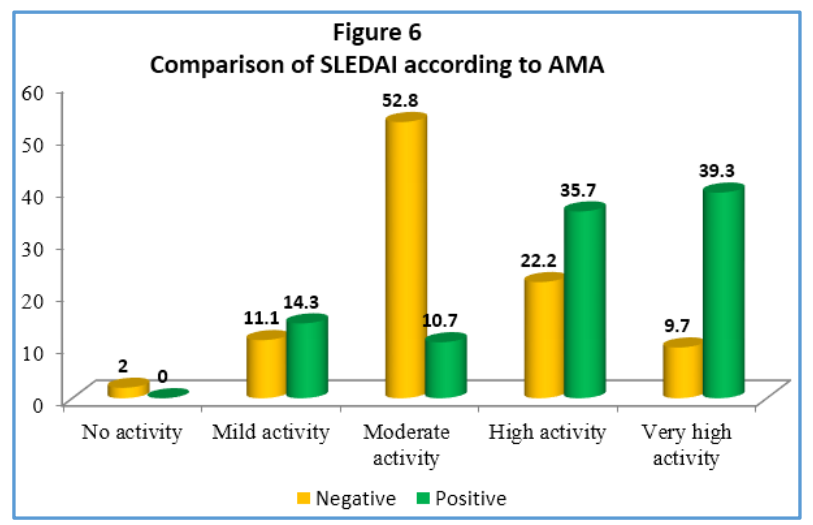

Mann-Whitney $\mathrm{U}$ test shows that AMA positivity and SLEDAI are closely related $(\mathrm{p}<0.01)$.

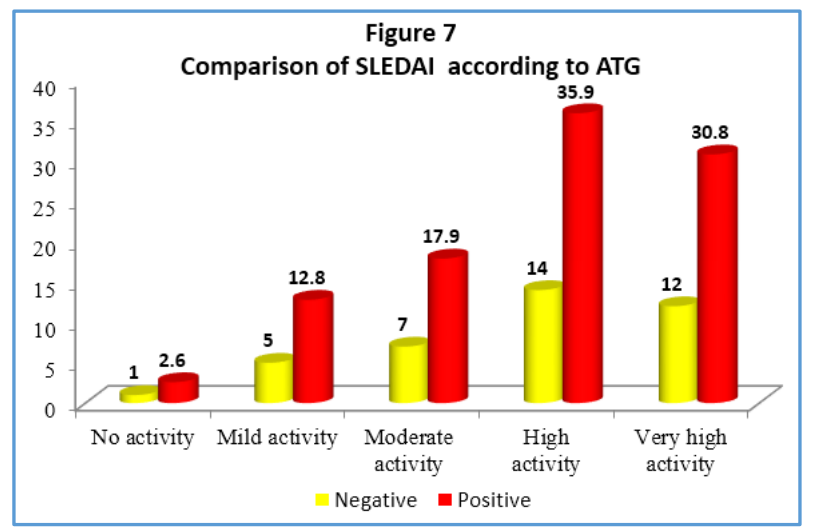

Mann-Whitney $U$ test shows that AMA positivity and SLEDAI are closely related $(\mathrm{p}<0.01)$. 
Fisher's exact test showed that proteinuria, lupus nephritis, vasculitis and fever in the SLEDAI are closely related to AMA positivity $(\mathrm{p}<0.05)$.

\section{DISCUSSION}

SLE is an autoimmune disorder often associated with other organ specific autoimmune disorders. The association between autoimmune thyroiditis, thyroid dysfunction and SLE has been reported in several studies and yielded conflicting conclusions. The prevalence of Antithyroid Antibodies (ATA) namely ATG and Anti-Thyroid Peroxidase (TPO) were reported to be higher in patients with SLE. Reported prevalence of autoimmune thyroid disease is (3.9 $24 \%$ ) and antithyroid antibodies is (11 - 51\%) in SLE patients. Clinical thyroid disease is more frequent in SLE patients than the normal population. ${ }^{8}$

100 patients with SLE admitted in general medicine were included in the study. These patients were assessed for the presence of thyroid dysfunction, thyroid antibodies and the association with SLEDAI. Of the 100 patients, 83\% were females and $17 \%$ were males. $41 \%$ were $<30$ yrs. of age $39 \%$ were in the 30 - 39 age group and $20 \%$ were above 40 yrs. of age.

Of the 100 patients $26 \%$ had hypothyroidism, $24 \%$ had subclinical hypothyroidism and $16 \%$ had hyperthyroidism. Kumar et $\mathrm{al}^{7}$ in his study has said 36\% lupus patients had thyroid dysfunction. In that study, primary hypothyroidism was the commonest dysfunction (14\%). This is similar to the study by Porkodi et al, ${ }^{9}$ in which twenty out of 153 patients had thyroid dysfunction. Subclinical hypothyroidism and hypothyroidism were the commonest dysfunction seen in $80 \%$ of patients. Miller et al ${ }^{10}$ in their study of 332 lupus patients revealed thyroid dysfunction in $7.5 \%$ with majority having hypothyroidism. Appenzeller et al11 in their study of 524 SLE patients concluded that there is a high prevalence of symptomatic as well as subclinical hypothyroidism and positive thyroid antibodies.

In our study of 100 patients, $28 \%$ were AMA positive and $39 \%$ were ATG positive. In the study by Porkadi ${ }^{9}$ et al, AMA was positive in $100 \%$ and ATG was positive in $82.4 \%$. Similar observations were made by Zakeri et al,12 which demonstrated higher levels of anti-TPO in SLE patients. Pyne et al in their study of 300 patients concluded that SLE patients had a prevalence of hypothyroidism greater than that of the normal population with a high frequency of both antimicrosomal and antithyroglobulin antibodies. Kausman et $\mathrm{al}^{13}$ in their study of 150 patients reported $21 \%$ were thyroid antibody positive.

In this study out of 100 patients, SLEDAI score showed $41 \%$ moderate activity, $26 \%$ high activity, $18 \%$ very high activity, $12 \%$ mild activity and $3 \%$ had no activity. The mean score was 12.6. In the study by Mader et al,14 out of 77 patients mean SLEDAI score was $5.48+$ + 5.41. In that study, levels of ATG/anti-TPO did not correlate with SLEDAI.

Among the clinical features arthritis was present in 73\%, $62 \%$ had malar rash, $27 \%$ had oral ulcers and $27 \%$ had serositis. Haematological manifestations were seen in $70 \%$ of patients.

ANA positivity was seen in $17 \%$ of patients, $79 \%$ were both ANA and anti-dsDNA positive and $2 \%$ were anti-dsDNA positive. There is a close association between thyroid autoantibody positivity and SLEDAI scoring $(\mathrm{p}<.01)$. There is increase in SLEDAI score with increasing values of antibody positivity.

Comparison of the clinical manifestations with thyroid antibodies was made. Lupus nephritis was present in $57.1 \%$ of AMA positive patients ( $\mathrm{p}<.01$ ), vasculitis in $21.4 \%$ of AMA positive $(\mathrm{p}<.01)$ and fever in $39.3 \%$ AMA positive $(\mathrm{p}<.05)$ Thus, lupus nephritis, vasculitis and fever in the SLEDAI are closely related to AMA positivity. When anti-thyroglobulin was compared, lupus nephritis was present in 51.3\%, vasculitis in $15.4 \%$ and fever in $35.9 \%$ of ATG associated (p< .05 ). Thus, lupus nephritis, vasculitis and fever were closely related to antimicrosomal and anti-thyroglobulin positivity ( $p$ $<.05)$.

\section{CONCLUSION}

In our present study, thyroid dysfunction was seen among SLE patients. Hypothyroidism was the major abnormality observed in this study. Positive thyroid auto-antibodies, both antimicrosomal and antithyroglobulin were found to be associated with SLE. There is a definite association between SLEDAI scores and positive thyroid antibodies. The SLEDAI Scores were found to be increasing with increasing values of thyroid antibody levels. In addition, there is a statistically significant association between lupus nephritis, vasculitis and positive thyroid antibody levels. Thus, from the present study, it is reasonable to conclude that thyroid autoantibodies may be an early marker of lupus nephritis and vasculitis. Further studies in larger population may be carried out to get a clearer understanding. This may help in early detection of organ damage and thereby prevent major complications.

\section{REFERENCES}

[1] Malaviya AN, Chandrasekaran AN, Kumar A, et al. Occasional series-lupus around the world systemic lupus erythematosus in India. Lupus 1997;6(9):690700 .

[2] Malaviya AN, Singh RR, Kumar A, et al. Systemic lupus erythematosus in northern India: a review of 329 cases. J Assoc Physicians India 1988;36(8):476-80.

[3] Hochberg MC. Updating the American college of rheumatology revised criteria for the classification of systemic lupus erythematosus. Arthritis Rheum 1997;40(9):1725.

[4] Symmons DP, Coppock JS, Bacon PA, et al. Development and assessment of a computerised index of clinical disease activity in systemic lupus erythematosus. Members of the British Isles lupus assessment group (BILAG). Q J Med 1988;69(259):927-37.

[5] Pyne D, Isenberg DA. Autoimmune thyroid disease in systemic lupus erythematosus. Ann Rheum Dis 2002;61(1):70-2.

[6] El-Sherif WT, El Gendi SS, Ashmawy MM, et al. Thyroid disorders and autoantibodies in systemic lupus erythematosus and rheumatoid arthritis patients. Egypt J Immunol 2004;11(2):81-90.

[7] Kumar K, Kole AK, Karmakar PS, et al. The spectrum of thyroid disorders in systemic lupus erythematosus. Rheumatology International 2012;32(1):73-8. 
[8] Rojas-Villarraga A, Toro C, Espinosa G, et al. Factors influencing poly autoimmunity in systemic lupus erythematosus. Autoimmunity Reviews 2010;9(4): 229-32.

[9] Madhavan R, Porkodi R, Ramakrishnnan S, et al. Systemic lupus erythematosis- the madras experience. J Assoc Physicians India 1983;36:481-4.

[10] Miller FW, Moore GF, Weintraub BD, et al. Prevalence of thyroid disease and abnormal thyroid function test results in patients with systemic lupus erythematosus. Arthritis Rheum 1987;30(10):1124-31.

[11] Appenzeller S, Pallone AT, Natalin RA, et al. Prevalence of thyroid dysfunction in systemic lupus erythematosus. J Clin Rheumatol 2009;15(3):117-9.
[12] Zakeri Z, Sandooghi M. Thyroid disorder in systemic lupus erythematosus patients in Southeast Iran. Shiraz E Medical Journal 2010;11(1):34-8.

[13] Kausman D, Isenberg DA. Thyroid autoimmunity in systemic lupus erythematosus: the clinical significance of a fluctuating course. British journal of Rheumatology 1995;34(4):361-4.

[14] Mader R, Mishail S, Adawi M, et al. Thyroid dysfunction in patients with systemic lupus erythematosus (SLE): relation to disease activity. Clin Rheumatol 2007;26(11):1891-4. 